\title{
LENGTHWISE FRACTURE ANALYSES OF FUNCTIONALLY GRADED BEAMS BY THE RAMBERG-OSGOOD EQUATION
}

\author{
Victor Rizov*
}

Department of Technical Mechanics, Faculty of Hydro-technique, University of Architecture, Civil Engineering and Geodesy, 1 Chr. Smirnesky blvd., 1046 - Sofia, Bulgaria

\begin{tabular}{l}
\hline ARTICLE INFO \\
\hline Article history: \\
Received: 26.10 .2017$. \\
Received in revised form: 21.01 .2018$. \\
Accepted: 05.02 .2018$. \\
\hline Keywords: \\
Functionally graded beam \\
Crack \\
Material non-linearity \\
\hline
\end{tabular}

DOI: https://doi.org/10.30765/er.38.3.8

\section{Introduction}

Functionally graded materials play a vital role in many engineering applications in aerospace, nuclear reactors, power plants, electronics, optics and

\begin{abstract}
:
The basic purpose of the present paper is to develop lengthwise fracture analyses of the functionally graded Symmetric Split Beam (SSB) configurations which exhibit non-linear mechanical behavior of the material. The SSB is loaded in pure bending. A lengthwise crack is located symmetrically with respect to the beam's mid-span. The crack is located arbitrary along the width of the beam's cross-section. Thus, the crack arms have different widths. The material is linearly and functionally graded along the height of the beam's crosssection. The material non-linearity is treated by the Ramberg-Osgood equation (this is one of the basic novelties introduced in this paper). The fracture is analyzed in terms of the strain energy release rate by applying three approaches. First, the strain energy release rate is derived by considering the balance of the energy. The strain energy release rate is obtained also by using the complementary strain energy. The fracture is analyzed also by the $J$-integral. The results obtained by the three approaches are identical which proves the correctness of the lengthwise fracture analyses developed in the present paper. A parametric study is carried-out in order to examine the influences of the material gradient, the lengthwise crack location along the beam's width, and the non-linear mechanical behavior of the functionally graded material on the fracture in the SSB configuration.
\end{abstract}

biomedicine. The basic advantage of the functionally graded materials over the traditional structural materials is the fact that the functionally graded materials permit spatial tailoring of the composition of their constituent materials during manufacturing

\footnotetext{
${ }^{*}$ Corresponding author. Tel.: + (359-2) 9635245 / 664; fax: + (359-2) 8656863

E-mail address: v_rizov_fhe@uacg.bg
} 
[1-17]. In this way, one can obtain maximum benefits from the in-homogeneity of the functionally graded materials. For instance, the behavior of functionally graded structural members and components to externally applied loadings and influences can be optimized.

The un-homogeneous character of the functionally graded materials makes the study of the mechanical response of the functionally graded structural members and components to the externally applied loading a challenging task [12-17]. An analytical approach for obtaining a solution for the stress state of a linear-elastic un-homogeneous cylinder with arbitrary varying material properties has been developed by applying a direct integration method [13]. It has been shown that the method allows for adequate modeling and analysis of mechanical behavior. An analytical solution for the stress state of a linear-elastic un-homogeneous rod subjected to external load which varies with axial coordinate has been derived [14]. It has been assumed that the material properties vary arbitrary in radial direction. The effect of the variable material properties on the stress distribution has been analyzed. The static buckling behavior of functionally graded shells has been studied assuming linear-elastic behavior of the material [16]. A new model of functionally graded thin-walled spherical shells loaded by external pressure has been applied to investigate the buckling problem [16].

One of the major concerns in the structural applications of functionally graded materials is their fracture behavior. Besides, the fracture analysis of functionally graded structures is more complicated in comparison to that of structures made of homogeneous materials. Recently, crack problems in functionally graded structures have received significant attention from research community around the world [18-20].

Fracture behavior of functionally graded three-point bending beam configurations has been studied in [18]. For this purpose, methods of linear-elastic fracture mechanics have been applied. By using the compliance approach, an equivalent homogeneous beam of variable height has been suggested for cracked three-point bending functionally graded beams. It has been shown that the equivalent beam captures the compliance characteristics of functionally graded beams. It has been concluded that the equivalent compliance concept can be extended to analyze other cracked functionally graded components loaded by concentrated forces.
Investigations of fracture behavior of functionally graded beams containing open edge cracks have been carried-out in [19]. It has been assumed that the material properties vary continuously along the height of the beam cross-section. The cracked sections of the beam have been modeled by using mass-less rotational springs. Continuity of the longitudinal and transverse displacements in the cracked sections has been assumed in the dynamic fracture analyses. Functionally graded beam configurations with clamped-free, hinged-hinged and clamped-clamped end supports have been investigated. Parametric studies have been conducted to show the effects of location and number of cracks on the flexural vibration and buckling behavior of linear-elastic functionally graded cracked beams.

The analyses of delamination fracture behavior of functionally graded beams with taking into account the material non-linearity have been developed in [20].

A solution for the strain energy release rate has been derived. The solution has been applied to investigate the delamination fracture behavior of the double cantilever beam configuration which is made of a functionally graded non-linear elastic material. It has been assumed that the material is functionally graded along the beam height. The non-linear mechanical behavior of the material has been described by using a power-law stress-strain relation.

This paper describes analyses of lengthwise fracture in functionally graded SSB configurations which exhibit non-linear mechanical behavior of the material. One of the most important novelties introduced in the present paper is the fact that the Ramberg - Osgood equation is used to deal with the non-linearity of the functionally graded material. Fracture is studied in terms of the strain energy release rate by considering the balance of energy. Influences of the material gradient along the beam height, the crack location along the beam width and the material non-linearity on the lengthwise fracture behavior are investigated.

\section{Lengthwise fracture analyses}

The present study is devoted to lengthwise fracture analyses of the functionally graded SSB configuration shown in Fig. 1. The beam has a rectangular cross-section of width, $b$, and height, $h$. The length of the beam is denoted by $2 l$. The external loading consists of two bending moments, $M$, applied at the free ends of the beam as shown in 
Fig. 1. A notch of depth, $b_{2}$, is introduced in the right-hand lateral surface of the beam in order to generate conditions for lengthwise fracture. It is assumed that a lengthwise crack of the length, $2 a$, is located symmetrically with respect to the beam midspan (it should be mentioned that one of the motives for the present study is the fact that functionally graded materials can be built up layer by layer [21], which is a premise for appearance of lengthwise cracks between layers [22, 23]). The lengthwise crack is located arbitrary along the width of the beam's cross-section (Fig.1). Thus, the two crack arms have different widths (the widths of the lefthand and the right-hand crack arms are denoted by $b_{1}$ and $b_{2}$, respectively).

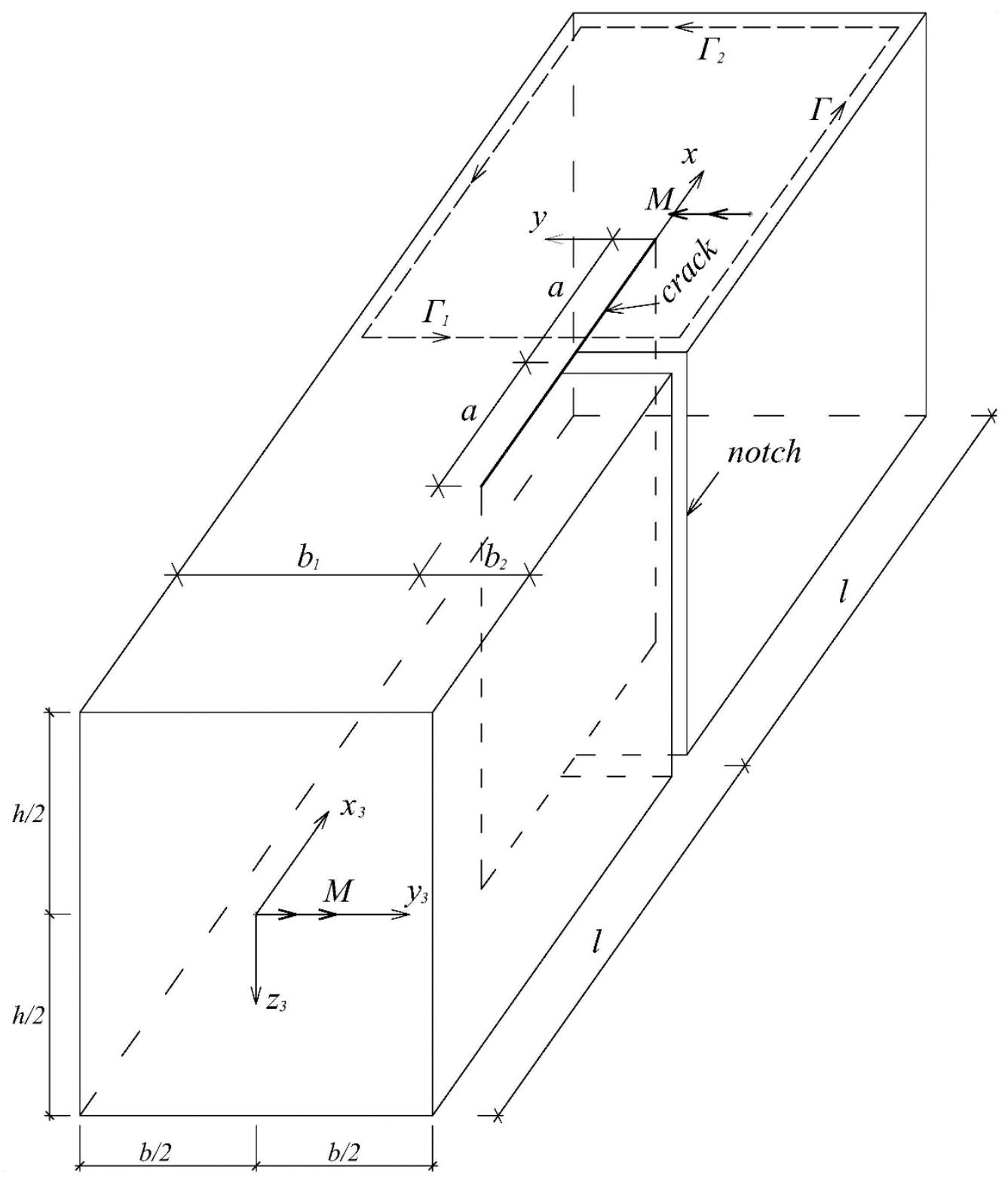

Figure 1. The geometry and loading of the functionally graded SSB configuration.

The left-hand crack arm has the following boundaries: $l-a \leq x_{3} \leq l+a,-b / 2 \leq y_{3} \leq b_{1}-b / 2$ and $-h / 2 \leq z_{3} \leq h / 2$. The boundaries of the righthand crack arm are $l-a \leq x_{3} \leq l+a$, $b_{1}-b / 2 \leq y_{3} \leq b / 2$ and $-h / 2 \leq z_{3} \leq h / 2$. The notch divides the right-hand crack arm in two symmetric segments of length, $a$, each. It is obvious 
that the segments of the right-hand crack arm are free of stresses (Fig. 1).

Due to the symmetry, only half of the beam, $l \leq x_{3} \leq 2 l$, is considered in the fracture analysis.

The functionally graded beam under consideration exhibits material non-linearity which is described by the Ramberg-Osgood stress-strain relation [24]

$$
\varepsilon=\frac{\sigma}{E}+\left(\frac{\sigma}{H}\right)^{\frac{1}{n}}
$$

where $\varepsilon$ is the longitudinal strain, $\sigma$ is the longitudinal normal stress, $E$ is the modulus of elasticity. The material properties, $H$ and $n$, in the second term of the right-hand part of formula (1) describe the material non-linearity.

It is assumed that the beam is made by a material which is functionally graded along the height of the beam cross-section. The modulus of elasticity varies continuously along the height the beam cross-section according to the following linear law:

$$
\begin{gathered}
E=E_{B}+\frac{E_{D}-E_{B}}{h}\left(\frac{h}{2}+z_{3}\right) \\
-\frac{h}{2} \leq z_{3} \leq \frac{h}{2}
\end{gathered}
$$

In (2), $E_{B}$ and $E_{D}$ are the values of the modulus of elasticity in the upper and lower surfaces of the beam, respectively.

The centroidal axis, $z_{3}$, is shown in Fig. 1.

The fracture behavior is analyzed in terms of the strain energy release rate, $G$. In order to derive the strain energy release rate, an increase of the crack length, $\delta a$, is assumed leading to an increase, $\delta \varphi$, of the rotation of the end section of the beam. The balance of the energy is written as

$$
M \delta \phi=\frac{\partial U}{\partial a} \delta a+G h \delta a
$$

where $U$ is the strain energy cumulated in half of the beam. Form (4), one derives

$$
G=2\left(\frac{M}{h} \frac{\partial \phi}{\partial a}-\frac{1}{h} \frac{\partial U}{\partial a}\right)
$$

It should be noted that the expression in brackets in (5) is doubled in view of the symmetry (Fig. 1). Formula (5) is used in the present paper to obtain the strain energy release rate. It should also be mentioned that the present analysis is valid for non-linear elastic behavior of the material. However, the analysis holds also for elastic-plastic behavior if the beam under consideration undergoes active deformation, i.e. if the external loading increases only [25, 26]. Also, the present analysis is developed by assuming validity of the hypothesis for small strains.

By applying methods of Mechanics of materials, the rotation of the end section of the beam is derived as

$$
\phi=a \kappa_{1}+(l-a) \kappa_{2}
$$

where $\kappa_{1}$ and $\kappa_{2}$ are, respectively, the curvatures of the left-hand crack arm and the un-cracked beam portion, $l+a \leq x_{3} \leq 2 l$, (Fig. 1).

The curvature of the left-hand crack arm is determined in the following way. First, the equations for equilibrium of the elementary forces in the crosssection of the left-hand crack arm are written as

$$
\begin{gathered}
N_{1}=b_{1} \int_{-\frac{h}{2}}^{\frac{h}{2}} \sigma d z_{1} \\
M_{y_{1}}=b_{1} \int_{-\frac{h}{2}}^{\frac{h}{2}} \sigma z_{1} d z_{1}
\end{gathered}
$$

where $z_{1}$ is the vertical centroidal axis of the crosssections of the left-hand crack arm (Fig. 2).

In (8), $N_{1}$ and $M_{y_{1}}$ are the axial force and the bending moment in the left-hand crack arm, respectively. It is obvious that (Fig. 1)

$$
N_{1}=0, M_{y_{1}}=M
$$

In order to determine $\kappa_{1}$ from equations (7) and (8), $z_{1}$ has to be expressed first as a function of $\sigma$ by using the Ramberg - Osgood stress-strain relation (1). The distribution of longitudinal strains in the beam under consideration is analyzed by applying the Bernoulli's hypothesis for plane sections since the beam has large span to height ratio. Concerning the 
distribution of longitudinal strains, it should also be mentioned that since the beam is loaded in pure bending (Fig. 1), the only non-zero strain is the longitudinal strain, $\varepsilon$. Thus, according to the small strain compatibility equations, $\varepsilon$ is distributed linearly along the height of the beam cross-section. Therefore, $\varepsilon$ is written as

$$
\varepsilon=\left(z_{1}-z_{1 n_{1}}\right) \kappa_{1}
$$

where $z_{1 n_{1}}$ is the coordinate of the neutral axis (Fig. 2). It should be noted that the neutral axis, $n_{1}-n_{1}$, shifts from the centroid since the material is functionally graded along the height of the beam's cross-section (Fig. 2).

The distribution of the modulus of elasticity along the height of the cross-section of the left-hand crack arm is expressed by (2). For thus purpose, $z_{3}$ is replaced with $z_{1}$ in (2). After substituting of (2) and (10) in (1), one obtains

$$
z_{1}=-\frac{\beta_{1}}{2 \beta_{2}}+\frac{z_{1 n_{1}}}{2}+\frac{\left(\frac{\sigma}{H}\right)^{\frac{1}{n}}}{2 \kappa_{1}}+\frac{\varphi}{2 \kappa_{1} \beta_{2}}
$$

where

$$
\begin{gathered}
\beta_{1}=\frac{E_{B}+E_{D}}{2} \\
\beta_{2}=\frac{E_{D}-E_{B}}{h} \\
\varphi=\sqrt{\left[\begin{array}{l}
\left.\kappa_{1} \beta_{1}-\kappa_{1} z_{1 n_{1}} \beta_{2}-\left(\frac{\sigma}{H}\right)^{\frac{1}{n}} \beta_{2}\right]^{2}+ \\
+4 \kappa_{1} \beta_{2}\left[\kappa_{1} z_{1 n_{1}} \beta_{1}+\sigma+\left(\frac{\sigma}{H}\right)^{\frac{1}{n}} \beta_{1}\right.
\end{array}\right]}
\end{gathered}
$$

By differentiating of (11) with respect to $\sigma$, one arrives at

$$
\begin{gathered}
d z_{1}=\frac{\sigma^{\frac{1-n}{n}}}{2 \kappa_{1} n H^{\frac{1}{n}}}+\frac{1}{2 \kappa_{1} \varphi n H^{\frac{2}{n}}}+ \\
+H^{\frac{1}{n}} \kappa_{1} \beta_{1} \sigma^{\frac{1-n}{n}} H^{\frac{1}{n}} \kappa_{1} z_{1 n_{1}} \beta_{2} \sigma^{\frac{1-n}{n}}+ \\
+\beta_{2} \sigma^{\frac{2-n}{n}}+ \\
+2 H^{\frac{2}{n}} \kappa_{1} n
\end{gathered}
$$

By substituting of (11) and (15) in (7) and (8) and integrating in boundaries from $\sigma_{B}$ and $\sigma_{D}$, where $\sigma_{B}$ and $\sigma_{D}$ are, respectively, the normal stresses in the upper and lower surfaces of the left-hand crack arm, one derives

$$
\begin{gathered}
N_{1}=b_{1}\left[\frac{\sigma_{D}^{\frac{1+n}{n}}-\sigma_{B}^{\frac{1+n}{n}}}{2 \kappa_{1}(1+n) H^{\frac{1}{n}}}+\frac{\sigma_{D}^{2}-\sigma_{B}^{2}}{2 \sqrt{\omega}}-\right. \\
\left.-\frac{2 \kappa_{1} \beta_{2}\left(\sigma_{D}^{3}-\sigma_{B}^{3}\right)}{3 \sqrt{\omega^{3}}}\right]
\end{gathered}
$$

$$
\left.\begin{array}{c}
M_{y_{1}}=b_{1}\left[\frac{\sigma_{D}^{\frac{1+2 n}{n}}-\sigma_{B}^{\frac{1+2 n}{n}}}{2 H^{\frac{1}{n}} \kappa_{1} \sqrt{\omega}(1+2 n)}+\right. \\
+\frac{\sigma_{D}^{\frac{1+n}{n}}-\sigma_{B}^{\frac{1+n}{n}}}{4 H^{\frac{1}{n}} \kappa_{1}(1+n)}\left(-\frac{\beta_{1}}{\beta_{2}}+z_{1 n_{1}}+\frac{\sqrt{\omega}}{\kappa_{1} \beta_{2}}\right)- \\
-\frac{\kappa_{1} \beta_{2}\left(\sigma_{D}^{4}-\sigma_{B}^{4}\right)}{2 \omega^{2}}+ \\
+\frac{\kappa_{1}\left(\sigma_{D}^{3}-\sigma_{B}^{3}\right)}{3 \omega^{\frac{3}{2}}\left(\beta_{1}-z_{1 n_{1}} \beta_{2}\right)+} \\
+\frac{\sigma_{D}^{2}-\sigma_{B}^{2}}{4}\left(-\frac{\beta_{1}}{\beta_{2} \sqrt{\omega}}+\frac{z_{1 n_{1}}}{\sqrt{\omega}}+\frac{1}{\kappa_{1} \beta_{2}}\right)
\end{array}\right]
$$




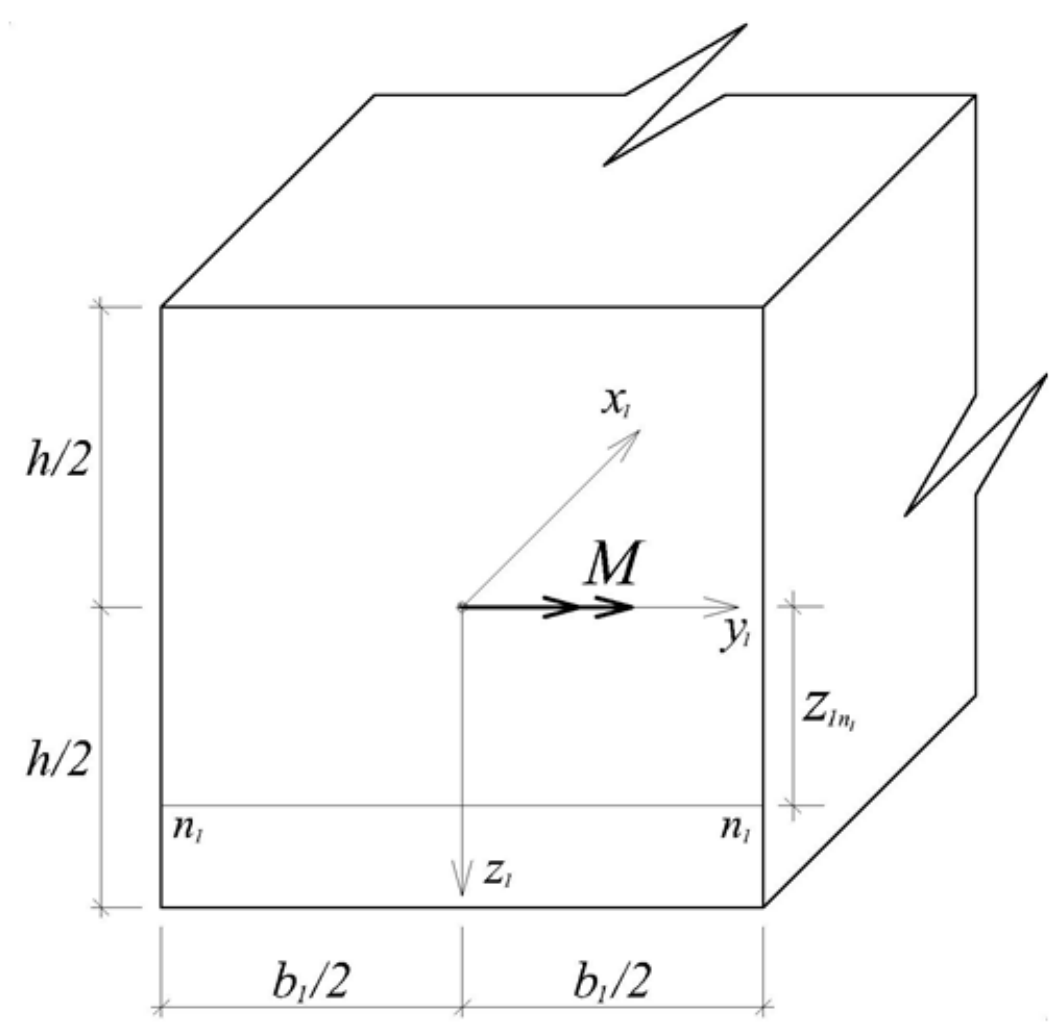

Figure 2. The cross-section of the left-hand crack arm in the beam mid-span.

where

$$
\omega=\left(\kappa_{1} \beta_{1}-\kappa_{1} z_{1 n_{1}} \beta_{2}\right)^{2}+4 \kappa_{1}^{2} \beta_{1} \beta_{2} z_{1 n_{1}}
$$

By using (1), (2) and (10), the following relations between $z_{1 n_{1}}, \kappa_{1}, \sigma_{B}$ and $\sigma_{D}$ are written:

$$
\begin{gathered}
\left(-\frac{h}{2}-z_{1 n_{1}}\right) \kappa_{1}=\frac{\sigma_{B}}{E_{B}}+\left(\frac{\sigma_{B}}{H}\right)^{\frac{1}{n}} \\
\left(\frac{h}{2}-z_{1 n_{1}}\right) \kappa_{1}=\frac{\sigma_{D}}{E_{D}}+\left(\frac{\sigma_{D}}{H}\right)^{\frac{1}{n}}
\end{gathered}
$$

Equations (16), (17), (19) and (20) should be solved with respect to $z_{1 n_{1}}, \kappa_{1}, \sigma_{B}$ and $\sigma_{D}$ by using the MATLAB computer program.

Equations (16), (17), (19) and (20) are used also to determine the neutral axis coordinate, $z_{2 n_{2}}$, the curvature, $\kappa_{2}$, of the un-cracked beam portion and the normal stresses in the upper and lower surfaces of the un-cracked beam portion, $\sigma_{B}^{u}$ and $\sigma_{D}^{u}$. For this purpose, $b_{1}, z_{1 n_{1}}, \kappa_{1}, \sigma_{B}$ and $\sigma_{D}$ are replaced with $b, z_{2 n_{2}}, \kappa_{2}, \sigma_{B}^{u}$ and $\sigma_{D}^{u}$, respectively.

Further, in order to derive the strain energy release rate by (5), the strain energy cumulated in half of the beam has to be determined. Since the two segments of the right-hand crack arm are free of stresses, the strain energy cumulated in half of the beam is written as

$$
U=U_{1}+U_{2}
$$

where $U_{1}$ and $U_{2}$ are the strain energies in the lefthand crack arm and the un-cracked beam portion, respectively.

$U_{1}$ is obtained by integrating the strain energy density, $u_{01}$, in the left-hand crack arm

$$
U_{1}=a b_{1} \int_{\sigma_{B}}^{\sigma_{D}} u_{01} d z_{1}
$$

where $d z_{1}$ is expressed as a function of $\sigma$ by (15). 
In principle, the strain energy density is equal to the area, $O P Q$, enclosed by the stress-strain curve (Fig. 3).

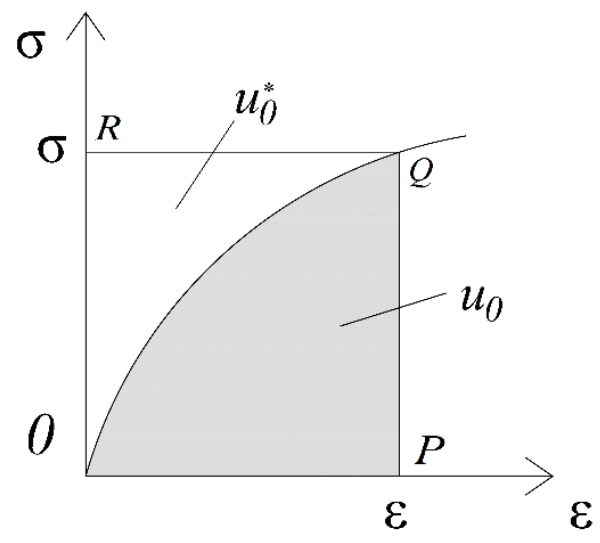

Figure 3. Schematic of a non-linear stress-strain curve (the strain energy and the complementary strain energy densities are denoted by $u_{0}$ and $u_{0}^{*}$, respectively).

For the Ramberg-Osgood stress-strain relation, the strain energy density is derived as [27]

$$
u_{01}=\frac{\sigma^{2}}{2 E}+\frac{\sigma^{\frac{1+n}{n}}}{(1+n) H^{\frac{1}{n}}}
$$

By substituting of (2), (12) and (13) in (23), one arrives at

$$
u_{01}=\frac{\sigma^{2}}{2\left(\beta_{1}+\beta_{2} z_{1}\right)}+\frac{\sigma^{\frac{1+n}{n}}}{(1+n) H^{\frac{1}{n}}}
$$

where $z_{1}$ is expressed as a function of $\sigma$ by (11).

The strain energy in the un-cracked beam portion is written as

$$
U_{2}=(l-a) b \int_{\sigma_{B}^{u}}^{\sigma_{D}^{u}} u_{02} d z_{2}
$$

where the strain energy density, $u_{02}$, is obtained by (24). For this purpose, $z_{1}, z_{1 n_{1}}$ and $\kappa_{1}$ are replaced, respectively, with $z_{2}, z_{2 n_{2}}$ and $\kappa_{2}$ in (11), (14), (15),
(24) and (25). Here, $z_{2}$ and $z_{2 n_{2}}$ are, respectively, the vertical centroidal axis and the coordinate of the neutral axis of the cross-section of the un-cracked beam portion. Formula (15) is applied to express $d z_{2}$ in (25) as a function of $\sigma$. For this purpose, $z_{1 n_{1}}$ and $\kappa_{1}$ are replaced, respectively, with $z_{2 n_{2}}$ and $\kappa_{2}$ in (14) and (15).

By substituting of (22) and (25) in (5), one arrives at

$$
\begin{gathered}
G=2\left[\frac{M}{h}\left(\kappa_{1}-\kappa_{2}\right)-\right. \\
\left.-\frac{b_{1}}{h} \int_{\sigma_{B}}^{\sigma_{D}} u_{01} d z_{1}+\frac{b}{h} \int_{\sigma_{B}^{u}}^{\sigma_{D}^{u}} u_{02} d z_{2}\right]
\end{gathered}
$$

The integration in (26) should be performed by the MATLAB computer program.

In order to verify (26), the strain energy release rate is derived also by considering the beam complementary strain energy. For this purpose, the strain energy release rate is written as [27]

$$
G=\frac{d U^{*}}{d A}
$$

where $U^{*}$ is the complementary strain energy cumulated in half of the beam, $A$ is the crack area. The elementary increase of the crack area is expressed as

$$
d A=h d a
$$

where $d a$ is an elementary increase of the crack length.

Since the segments of the right-hand crack arm are free of stresses, the complementary strain energy cumulated in half of the beam is written as

$$
U^{*}=U_{1}^{*}+U_{2}^{*}
$$

strain energies in the left-hand crack arm and the uncracked beam portion, respectively.

The complementary strain energy cumulated in the left-hand crack arm is written as

$$
U_{1}^{*}=a b_{1} \int_{\sigma_{B}}^{\sigma_{D}} u_{01}^{*} d z_{1}
$$


where $u_{01}^{*}$ is the complementary strain energy density in the left-hand crack arm. In (30), $d z_{1}$ is expressed as a function of $\sigma$ by (15).

The complementary strain energy density is equal to the area, $O Q R$, which supplements the area, $O P Q$, enclosed by the stress-strain curve, to a rectangle (Fig. 3). Thus, $u_{01}^{*}$ is expressed as

$$
u_{01}^{*}=\sigma \varepsilon-u_{01}
$$

By combining of (1), (2), (12), (13), (24) and (31), one arrives at

$$
u_{01}^{*}=\frac{\sigma^{2}}{2\left(\beta_{1}+\beta_{2} z_{1}\right)}+\frac{n \sigma^{\frac{1+n}{n}}}{(1+n) H^{\frac{1}{n}}}
$$

The complementary strain energy cumulated in the un-cracked beam portion is written as

$$
U_{2}^{*}=(l-a) b \int_{\sigma_{B}^{u}}^{\sigma_{D}^{u}} u_{02}^{*} d z_{2}
$$

where the complementary strain energy density, $u_{02}^{*}$ in the un-cracked beam portion is found by (32). For this purpose, $z_{1}, z_{1 n_{1}}$ and $\kappa_{1}$ are replaced, respectively, with $z_{2}, z_{2 n_{2}}$ and $\kappa_{2}$ in (11), (14), (15), and (32). Also, formula (15) is applied to express $d z_{2}$ in (33) as a function of $\sigma$. For this purpose, $z_{1 n_{1}}$ and $\kappa_{1}$ are replaced, respectively, with $z_{2 n_{2}}$ and $\kappa_{2}$ in (14) and (15).

The expression obtained by substituting of (28), (29), (30) and (33) in (27) is doubled in view of the symmetry (Fig. 1)

$$
G=\frac{2}{h}\left(b_{1} \int_{\sigma_{B}}^{\sigma_{D}} u_{01}^{*} d z_{1}-b \int_{\sigma_{B}^{u}}^{\sigma_{D}^{u}} u_{02}^{*} d z_{2}\right)
$$

Integration in (34) should be performed by the MATLAB computer program. It should be noted that the strain energy release rates derived by (34) matches exactly the strain energy release rates obtained by (26). This fact is a verification of the lengthwise fracture analysis of the functionally graded SSB configuration performed in the present paper.

The solution for the strain energy release rate (26) is compared also with the $J$-integral approach [28]. The $J$-integral is solved along the integration contour, $\Gamma$, shown by a dashed line in Fig. 1. Obviously, the $J$ integral has non-zero values only in segments, $\Gamma_{1}$ and $\Gamma_{2}$, of the integration contour $\left(\Gamma_{1}\right.$ and $\Gamma_{2}$ coincide with the cross-section of the left-hand crack arm in the beam mid-span and the end section of the beam, respectively).

The $J$-integral in segment, $\Gamma_{1}$, of the integration contour is written as

$$
J_{1}=\int_{\Gamma_{1}}\left[u_{01} \cos \alpha_{1}-\left(p_{x_{1}} \frac{\partial u}{\partial x_{1}}+p_{y_{1}} \frac{\partial v}{\partial x_{1}}\right)\right] d s_{1}
$$

where $\alpha_{1}$ is the angle between the outwards normal vector to the contour of integration and the crack direction, $p_{x_{1}}$ and $p_{y_{1}}$ are the components of the stress vector, $u$ and $v$ are the components of the displacement vector with respect to the coordinate system $x y$, and $d s_{1}$ is a differential element along the contour of integration.

The components of (35) are determined as

$$
\begin{gathered}
p_{x_{1}}=-\sigma \\
p_{y_{1}}=0 \\
d s_{1}=d y_{1} \\
\cos \alpha_{1}=-1
\end{gathered}
$$

In (38), the coordinate, $y_{1}$, varies in the interval $\left[-b_{1} / 2 ; b_{1} / 2\right]$.

The partial derivative, $\partial u / \partial x_{1}$, in (35) is obtained by using the following formula from Mechanics of materials:

$$
\frac{\partial u}{\partial x_{1}}=\varepsilon
$$

By using (1), (2), (12) and (13), one obtains 


$$
\varepsilon=\frac{\sigma}{\beta_{1}+\beta_{2} z_{1}}+\left(\frac{\sigma}{H}\right)^{\frac{1}{n}}
$$

where $z_{1}$ is expressed as a function of $\sigma$ by (11).

By substituting of (36), (37), (38), (39), (40) and (41) in (35), one derives

$$
J_{1}=-b_{1} u_{01}+b_{1}\left(\frac{\sigma^{2}}{\beta_{1}+\beta_{2} z_{1}}+\frac{\sigma^{\frac{1+n}{n}}}{H^{\frac{1}{n}}}\right)
$$

In (42), $u_{01}$ is obtained by (24) where $z_{1}$ is expressed as a function of $\sigma$ by (11).

The $J$-integral in segment, $\Gamma_{2}$, is written as

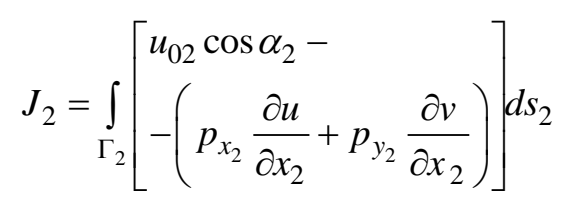

The components of (43) are obtained as

$$
\begin{gathered}
p_{x_{2}}=\sigma \\
p_{y_{2}}=0 \\
d s_{2}=-d y_{2} \\
\cos \alpha_{1}=1 \\
\frac{\partial u}{\partial x_{2}}=\frac{\sigma}{\beta_{1}+\beta_{2} z_{2}}+\left(\frac{\sigma}{H}\right)^{\frac{1}{n}}
\end{gathered}
$$

The coordinate, $y_{2}$, varies in the interval $[-b / 2 ; b / 2]$. The coordinate, $z_{2}$, is expressed as function of $\sigma$ by (11). For this purpose, $z_{1 n_{1}}$ and $\kappa_{1}$ are replaced, respectively, with $z_{2 n_{2}}$ and $\kappa_{2}$ in (11) and (14).

By substituting of (44), (45), (46), (47) and (48) in (43), one derives

$$
J_{2}=b u_{02}-b\left(\frac{\sigma^{2}}{\beta_{1}+\beta_{2} z_{2}}+\frac{\sigma^{\frac{1+n}{n}}}{H^{\frac{1}{n}}}\right)
$$

The average value of the $J$-integral along the front of the lengthwise crack is written as

$$
J=\frac{1}{h}\left(\int_{\sigma_{B}}^{\sigma_{D}} J_{1} d z_{1}+\int_{\sigma_{B}^{u}}^{\sigma_{D}^{u}} J_{2} d z_{2}\right)
$$

By doubling (in view of the symmetry) of the expression obtained after substituting of (42) and (49) in (50), one arrives at

$$
\begin{aligned}
& J=\frac{2}{h}\left\{\int _ { \sigma _ { B } } ^ { \sigma _ { D } } \left[-b_{1} u_{01}+\right.\right. \\
& \left.+b_{1}\left(\frac{\sigma^{2}}{\beta_{1}+\beta_{2} z_{1}}+\frac{\sigma^{\frac{1+n}{n}}}{H^{\frac{1}{n}}}\right)\right] d z_{1}+ \\
& +\int_{\sigma_{B}^{u}}^{\sigma_{D}^{u}}\left[b u_{02}-\right. \\
& \left.\left.-b\left(\frac{\sigma^{2}}{\beta_{1}+\beta_{2} z_{2}}+\frac{\sigma^{\frac{1+n}{n}}}{H^{\frac{1}{n}}}\right)\right] d z_{2}\right\}
\end{aligned}
$$

where $d z_{1}$ is expressed as a function of $\sigma$ by (15). Formula (15) is used also to express $d z_{2}$ as a function of $\sigma$. For this purpose, $z_{1 n_{1}}$ and $\kappa_{1}$ are replaced, respectively, with $z_{2 n_{2}}$ and $\kappa_{2}$ in (14) and (15).

Integration in (51) should be performed by the MATLAB computer program. It should be noted that the $J$-integral values obtained by (51) are exact match of the strain energy release rates calculated by (26), which also verifies the lengthwise fracture analysis developed in the present paper.

\section{Parametric studies}

Parametric studies of lengthwise fracture in the functionally graded SSB configuration (Fig.1) are carried-out in order to investigate the effects of material gradient, crack location along the beam width and material non-linearity on the fracture 
behavior. Fracture is analyzed in terms of the strain energy release rate by applying formula (26).

The calculated strain energy release rate is presented in non-dimensional form by using the formula $G_{N}=G /\left(E_{B} h\right)$. The location of the lengthwise crack along the beam width is characterized by $b_{1} / b$ ratio. The $E_{D} / E_{B}$ ratio is used to characterize the material gradient along the beam height. The calculations are performed assuming that $b=0.012$ $\mathrm{m}, h=0.018 \mathrm{~m}, n=0.7$ and $M=25 \mathrm{Nm}$. The strain energy release rate in non-dimensional form is presented as a function of $H / E_{B}$ ratio at $E_{D} / E_{B}=0.5$ in Fig. 4 for three $b_{1} / b$ ratios.

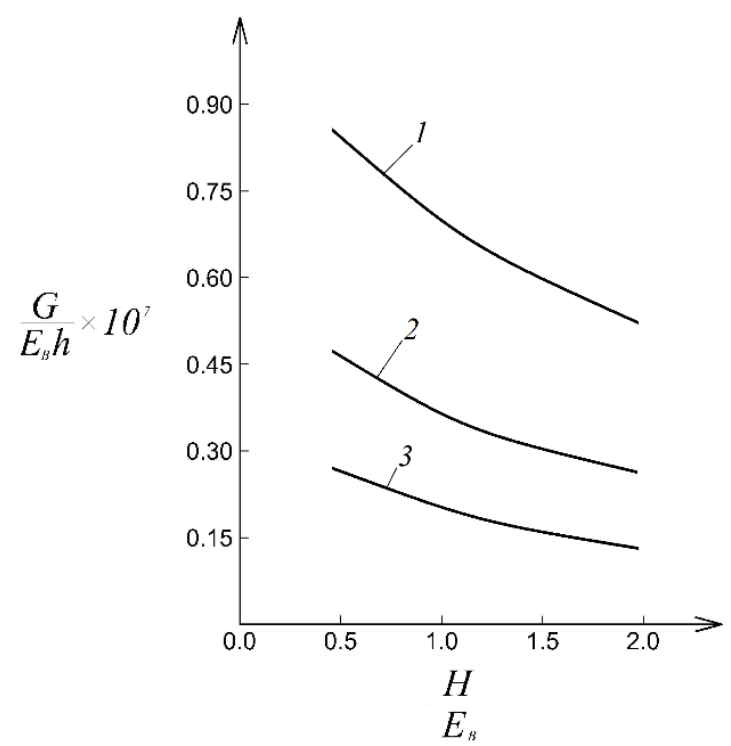

Figure 4. The strain energy release rate in nondimensional form plotted against $H / E_{B}$ ratio at $b_{1} / b=0.25$ (curve 1 ),

$b_{1} / b=0.50$ (curve 2) and $b_{1} / b=0.75$ (curve 3).

One can observe in Fig. 4 that the strain energy release rate decreases with increasing of $H / E_{B}$ ratio. The curves in Fig. 4 show also that the strain energy release rate decreases when $b_{1} / b$ ratio increases. This finding is attributed to the fact the stiffness of the left-hand crack arm increases with increasing of $b_{1} / b$ ratio.

The influence of the material gradient along the beam height on the lengthwise fracture behavior is investigated too. For this purpose, the strain energy release rate in non-dimensional form is plotted against $E_{D} / E_{B}$ ratio at $H / E_{B}=1.4$ and $b_{1} / b=0.75$ in Fig. 5 . It should be noted that $E_{B}$ is kept constant in the calculations. Therefore, $E_{D}$ is varied in order to obtain various $E_{D} / E_{B}$ ratios. It can be observed in Fig. 5 that the increase of $E_{D} / E_{B}$ ratio leads to decrease of the strain energy release rate (the reason for this behavior is the increase of the beam stiffness with increasing of $E_{D} / E_{B}$ ratio).

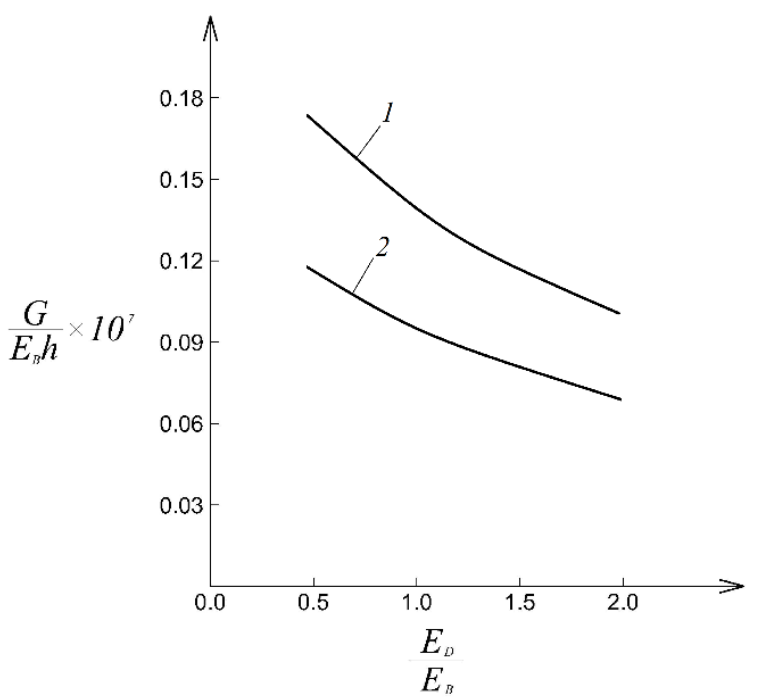

Figure 5. The strain energy release rate in nondimensional form plotted against $E_{D} / E_{B}$ ratio at non-linear (curve 1) and linearelastic (curve 2) behavior of the functionally graded material.

The effect of material non-linearity on the strain energy release rate is also evaluated. For this purpose, the strain energy release rate derived assuming linear-elastic behavior of the functionally graded beam is plotted also in Fig. 5 for comparison with the non-linear solution. It should be mentioned that the linear-elastic solution for the strain energy release rate is obtained by substituting of $H \rightarrow \infty$ in formula (26), which follows from the fact at $H \rightarrow \infty$, the Ramberg - Osgood stress-strain relation transforms into the Hooke's law. The diagrams in Fig. 5 indicate that the material nonlinearity leads to increases of the strain energy release rate.

\section{Conclusions}

Lengthwise fracture in functionally graded SSB configurations which exhibit non-linear mechanical 
behavior of the material is analyzed. The material non-linearity is described by applying the RambergOsgood stress-strain relation. The SSB configurations with a lengthwise crack located arbitrary along the beam width are investigated. Thus, the solution obtained can be used to evaluate the influence of crack location on the lengthwise fracture behavior. The material is functionally graded along the height of the beam cross-section (a linear law is applied in order to describe the continuous variation of the modulus of elasticity along the height of the beam cross-section). The fracture behavior is analyzed in terms of the strain energy release rate by considering the balance of energy. Comparisons with solution for the strain energy release rate obtained by using the beam complementary strain energy and with lengthwise fracture analysis by the $J$-integral approach are carried-out for verification. The effects of material gradient, crack location along the beam width and the material non-linearity on the lengthwise fracture behavior of the functionally graded SSB configuration are investigated. The most important findings from the lengthwise fracture analyses developed in the present paper can be summarized as follows:

- The strain energy release rate decreases with increasing $H / E_{B}$ ratio.

- The increase of the width of the cross-section of the left-hand crack arm leads to decrease of the strain energy release rate.

- The strain energy release rate decreases when $E_{D} / E_{B}$ ratio increases.

\section{References}

[1] Hirai, T., Chen, L.: Recent and prospective development of functionally graded materials in Japan, Mater Sci. Forum, 308-311(1999), 2, 509-514.

[2] Lu, C. F., Lim, C. W., Chen, W. Q.: Semianalytical analysis for multi-dimensional functionally graded plates: 3-D elasticity solutions, Int. J. Num. Meth. Engng, 79(2009), 3, 25-44.

[3] Markworth, A. J., Ramesh, K. S., Parks, Jr. W. P.: Review: modeling studies applied to functionally graded materials, J. Mater. Sci., 30(1995), 3, 2183-2193.

[4] Nemat-Allal, M. M., Ata, M. H., Bayoumi, M. R. Khair-Eldeen, W.: Powder metallurgical fabrication and microstructural investigations of Aluminum/Steel functionally graded material,
Materials Sciences and Applications, 2(2011), 2, 1708-1718.

[5] Gasik, M. M.: Functionally graded materials: bulk processing techniques, International Journal of Materials and Product Technology, 39(2010), 4, 20-29.

[6] Butcher, R. J., Rousseau, C. E., Tippur, H. V.: A functionally graded particulate composite: Measurements and Faiure Analysis, Acta Matererialia, 47(1999), 1, 259-268.

[7] Bykov, Yu. V., Egorov, S. V., Ermeev, A. G., Holoptsev, V. V.: Fabrication of metal-ceramic functionally graded materials by microwave sintering, Inorganic Materials: Applied Research 3(2012), 3, doi: 10.1134 / S20751133120300057.

[8] Lu, C. F., Lim, C. W., Chen, W. Q.: Semianalytical analysis for multi-dimensional functionally graded plates: 3-D elasticity solutions, Int. J. Num. Meth. Engng, 79(2009), 5, 25-44.

[9] Koizumi, M.: The concept of FGM Ceramic Trans., Functionally Gradient Materials, 34(1993), 3, 3-10.

[10] Suresh, S., Mortensen, A.: Fundamentals of functionally graded materials. IOM Communications Ltd, London, 1998.

[11] Prasad, P.: Design and Analysis of a Disc Brake by Using Functional Graded Materials, International Journal of Research, 4(2017), 6, 103-110.

[12] Tokovyy, Y., Ma, C.-C.: Three-Dimensional Temperature and Thermal Stress Analysis of an Inhomogeneous Layer, Journal of Thermal Stresses, 36(2013), 2, 790 - 808 DOI: 10.1080/01495739.2013.787853.

[13] Tokova, L., Yasinskyy, A., Ma, C.-C.: Effect of the layer in homogeneity on the distribution of stresses and displacements in an elastic multilayer cylinder, Acta Mechanica, (2016), DOI: 10.1007/s00707-015-1519-8, 1 - 13.

[14] Tokovyy, Y., Ma, C.-C.: Axisymmetric Stresses in an Elastic Radially Inhomogeneous Cylinder Under Length-Varying Loadings, ASME Journal of Applied Mechanics, 83(2016): DOI: 10.1115/1.4034459.

[15] Uslu Uysal, M., Kremzer, M.: Buckling Behaviour of Short Cylindrical Functionally Gradient Polymeric Materials, Acta Physica Polonica A, 127 (2015), 2, 1355-1357, DOI:10.12693/APhysPolA.127.1355.

[16] Uslu Uysal, M.: Buckling behaviours of functionally graded polymeric thin-walled 
hemispherical shells. Steel and Composite Structures, An International Journal, 21 (2016), 1, 849-862.

[17] Uslu Uysal, M., Güven, U.: A Bonded Plate Having Orthotropic Inclusion in Adhesive Layer under In-Plane Shear Loading, The Journal of Adhesion, 92 (2016), 2, 214-235, DOI:10.1080/00218464.2015.1019064.

[18] Upadhyay, A. K., Simha, K. R. Y.: Equivalent homogeneous variable depth beams for cracked FGM beams; compliance approach, International Journal of Fracture, 144(2007), 4, 209-213.

[19] Yang, J., Chen, Y.: Free vibration and buckling analyses of functionally graded beams with edge cracks, Composite Structures, 83(2008), 3, 48 60.

[20] Rizov, V.: An analytical solution to the strain energy release rate of a crack in functionally graded beams, European journal of mechanics A/solids, 65(2017), 6, 301-312.
[21] Bohidar, S. K., Sharma, R., Mishra, P. R.: Functionally graded materials: A critical review, International Journal of Research, 1(2014), 4, 289-301.

[22] Dolgov, N. A.: Determination of Stresses in a Two-Layer Coating, Strength of Materials, 37(2005), 4, 422-431.

[23] Dolgov, N. A.: Analytical Methods to Determine the Stress State in the Substrate-Coating System Under Mechanical Loads, Strength of Materials, 48(2016), 5, 658-667.

[24] Dowling, N.: Mechanical Behavior of Materials. Pearson, 2007.

[25] Chakrabarty, J.: Theory of plasticity. Elsevier Butterworth-Heinemann, Oxford, 2006.

[26] Lubliner, J.: Plasticity theory (Revised edition). University of California, Berkeley, CA, 2006.

[27] Rizov, V.: Delamination analysis of a layered elastic-plastic beam, International Journal of Structural Integrity, 8(2017), 4, 516-529.

[28] Broek, D.: Elementary engineering fracture mechanics. Springer, 1986. 